\title{
Incomplete sums of non-negative multiplicative functions
}

\author{
by \\ George Greaves (Cardiff)
}

1. Introduction. Let $g$ be multiplicative, and suppose that $g(n) \geq 0$ whenever $n \geq 1$. In this paper we also suppose $g(n)=0$ if $n$ is not squarefree. For specified $\kappa>0$ define $\eta$ by the equation

$$
\sum_{p<v} g(p) \log p=\kappa \log v+\eta(v) \quad \text { if } v \geq 1 .
$$

In this paper we shall assume the existence of a constant $A$ such that

$$
\eta(v)-\eta(u) \leq A \quad \text { when } 1<u<v .
$$

In particular, (1.2) applies if $g(n)$ is of size "about $1 / n$ "; it could be $\kappa^{\omega(n)} / n$, for example. A convenient convention will be

$$
\eta(v)=0 \quad \text { if } v<1,
$$

which does not conflict with (1.2).

Multiplicative functions of this type arise, for example, in applications of Selberg's sieve method, where the object of interest is the sum

$$
G_{z}(x)=\sum_{\substack{1 \leq n<x \\ p \mid n \Rightarrow p<z}} g(n) .
$$

In the more straightforward applications of the sieve only the case $z=x$ need arise, but in the more involved parts of the theory it is necessary to consider the case when $z<x$. Thus the sum is over integers which it is now customary to refer to as "smooth", or occasionally as "friable", the terminology "incomplete" for sums of this type being an older one.

In terms of the function $g,(1.2)$ says

$$
\sum_{u \leq p<v} g(p) \log p \leq \kappa \log \frac{v}{u}+A \quad \text { if } 1<u<v .
$$

2000 Mathematics Subject Classification: Primary 11N37; Secondary 11N35. 
In the context of the sieve method, situations arise (cf. $§ 1.3 .5$ in [3], for example) in which the readily available information about the function $g$ is of this form. Theorem 1 below is concerned with the estimation of the sum $G_{z}(x)$ in this context.

In work on multiplicative functions it is more usual to make a two-sided assumption in place of (1.2), for example

$$
|\eta(v)| \leq \frac{1}{2} A \quad \text { when } v \geq 1 .
$$

This implies the two-sided version of (1.2), in which the left side is replaced by its absolute value. The analogous two-sided conclusion appears in Theorem 2 , where we also assume that the constant $A$ can be chosen so that

$$
\sum_{p<z} g^{2}(p) \log p \leq A
$$

as is straightforward when the series $\sum g^{2}(p) \log p$ converges.

Write $x=z^{s}$. A result of Song [11] implies that when $0<\varepsilon<1$, and on the basis of an assumption slightly weaker than (1.5),

$$
\frac{G_{z}\left(z^{s}\right)}{G_{z}(\infty)}=\sigma(s)+O\left(\frac{\log (s+1)}{(\log z)^{1-\varepsilon}}\right),
$$

for a certain function $\sigma(s)$ which we describe below. The factor $\log (s+1)$ was promptly removed by Tenenbaum in [13]. Note that the apparent convergence question implied by the symbol $\infty$ is a trivial one, since the sum in (1.4) is independent of $x$ as soon as $x$ is large enough. Here, and in (1.8) below, the $O$-constant may depend on $\varepsilon$ and on the constants $A$ and $\kappa$ from (1.1), (1.5) and (1.6). Elsewhere, however, $O$-constants will depend only on $\kappa$, their dependence on $A$ being made explicit.

Halberstam and Richert had previously obtained a result like the case $\varepsilon=0$ of (1.7) in which the increasing factor $\log (s+1)$ assumed the weaker form $s^{2 \kappa+1}$. Song's paper used additional ideas drawn from a paper of Hildebrand $[8]$ on the related but more difficult sum

$$
\Psi(x, z)=\sum_{\substack{1 \leq n<x \\ p \mid n \Rightarrow p<z}} 1,
$$

for which Hildebrand noted that a corresponding factor $\log (s+1)$ cannot be improved. Actually Song's result was obtained in a more general context than that just described. In particular our assumption that $g\left(p^{\alpha}\right)=0$ at prime powers $p^{\alpha}$ when $\alpha \geq 2$ can be appreciably relaxed. We do not examine this situation in this article, but Theorem 1 relaxes the condition (1.5) in a different respect. Generalisation in other directions is also possible, an account of which may appear in due course. 
The formula (1.7) is, however, rather weak in its $s$-aspect. The continuous expression $\sigma(s)$ satisfies $\sigma(s)=1+O\left(e^{-s \log s}\right)$, and Lemma 1 (below) implies

$$
\frac{G_{z}\left(z^{s}\right)}{G_{z}(\infty)}=1-e^{-s \log s+O(s)} .
$$

The comparatively weak nature of the $s$-dependence of the $O$-term in (1.7) is therefore somewhat disappointing.

In this article we describe a method which allows us to combine the $z$-dependence in (1.7) with the $s$-dependence from (1.8).

Our result is enunciated in terms of the following notation. The continuous function $\sigma$ can be defined by requiring

$$
\sigma(s)=0 \quad \text { if } s<0, \quad \frac{d}{d s}\left(\frac{\sigma(s)}{s^{\kappa}}\right)+\frac{\kappa \sigma(s-1)}{s^{\kappa+1}}=0 \quad \text { if } s \neq 0,
$$

so that $\sigma(s)=C s^{\kappa}$ when $0 \leq s \leq 1$, for some constant $C$ : we specify

$$
C=e^{-\gamma \kappa} / \Gamma(\kappa+1) \text {. }
$$

For larger $s$ the function $\sigma(s)$ is defined on $[0, N]$ by induction on $N$, using successive integrations of (1.9). The choice of $C$ gives (see [1], or $\S 7.1 .1$ in [3] for details) $\sigma(s) \rightarrow 1$ as $s \rightarrow \infty$. We will say more about the function $\sigma$ later. The notation $\sigma$ is not exactly as in either [6] or [11].

A simple example of this situation is when $g(p)=1 / \phi(p), \phi$ being Euler's totient function. In this case (1.5) holds when $\kappa=1$, and at $s=1$ (1.7) reduces to the well known estimate

$$
\sum_{1 \leq n<x} \frac{|\mu(n)|}{\phi(n)}=\log x+O(1) .
$$

In the general case, let $B$ be any number for which

$$
B \geq 1, \quad B(z):=\frac{1}{\log z} \sum_{p<z} \frac{g(p)}{1+g(p)} \log p \leq B .
$$

We suppose $B \geq 1$ for convenience, and because our Lemma 1 is rather weak for small $B$. Then define

$$
\psi_{B}(v)=\int_{B<t<v} \log \frac{t}{B} d t= \begin{cases}v \log \frac{v}{B}-v+B & \text { if } v>B, \\ 0 & \text { otherwise. }\end{cases}
$$

If (1.2) or (1.5) holds then (1.11) follows with $B=\kappa+A / \log z$, and hence with $B=\kappa+A / \log 2$. In the treatment of the ensuing theorems we weaken this statement to $B=O(A)$.

TheOREM 1. Suppose that $g$ is multiplicative, that $g(n) \geq 0$, and that $g\left(p^{\alpha}\right)=0$ when $\alpha \geq 2$ and $p$ is prime. Assume that $\kappa>0$ and $A \geq 1$ 
are such that (1.2) holds when $v<z$. Then the sum $G_{z}\left(z^{s}\right)$ defined in (1.4) satisfies

$$
\frac{G_{z}\left(z^{s}\right)}{G_{z}(\infty)} \geq \sigma(s)-\frac{e^{-\psi_{B}(s)+O(A)}}{\log z} \quad \text { when } s \geq 1,
$$

in which the O-constant depends only upon $\kappa$.

The sense of the inequality in Theorem 1 reflects the fact, noted and used in [9], that the quotient on the left increases if an individual value of $g(p)$ is decreased.

If we are not concerned with the particular requirements of the sieve method and its applications then it is more usual to draw a two-sided conclusion on the basis of a corresponding two-sided hypothesis. The method used here leads to the following two-sided analogue of Theorem 1.

TheOREM 2. Let $g$ be as in Theorem 1, but suppose that $\kappa>0$ and $A \geq 1$ are such that (1.6) applies and (1.5) holds when $v<z$. Then the sum $G_{z}\left(z^{s}\right)$ defined in (1.4) satisfies

$$
\left|\frac{G_{z}\left(z^{s}\right)}{G_{z}(\infty)}-\sigma(s)\right| \leq \frac{s e^{-\psi_{B}(s)+O(A)}}{\log z} \quad \text { when } s \geq 1,
$$

in which the implied constant depends only upon $\kappa$, and $\sigma$ is as in (1.9).

Actually Theorem 2 is rather weak in its upper-bound aspect. Because $G_{z}\left(z^{s}\right) \leq G_{z}(\infty)$, the upper bound for $G_{z}\left(z^{s}\right)$ implied by Theorem 2 becomes trivial when the entry on the right exceeds $1-\sigma(s)$. Actually an asymptotic analysis shows $1-\sigma(s)=e^{-s \log s-s \log \log s+O(s)}$ (see [7] for a much stronger result), so this triviality has already arisen when $s>\log \log z$, for example. Recently, developing on further work of Song [12], G. Tenenbaum and $\mathrm{J}$. Wu [14] have studied the related but harder questions in which, in essence, the summand $g(n)$ is replaced by $n g(n)$, on the basis of a stronger assumption about $g(p)$ involving $\sum p g(p) \log p$ rather than $\sum g(p) \log p$. After seeing a preliminary draft of this paper, Tenenbaum pointed out to the author that a partial summation based on Theorem 2.1 in [14] will lead (on the basis of the stronger assumption about $g(p))$ to a version of Theorem 2 in which a factor $1-\sigma(s)$ appears on the right instead of the exponential in Theorems 1 and 2, and which is therefore appreciably stronger for large $s$.

An attempt at a corresponding inference in connection with Theorem 1 would, however, involve further considerations.

The method adopted in this paper is of a rather different character. In particular, we make use of an estimate (provided in Lemma 1) depending only on the rather simple device attributed to R. A. Rankin, in contrast to the more analytic methods used in [14]. Any weakness in this estimate does, of course, lead to a corresponding weakness in our Theorems 1 and 2. 
The methods employed in [6], and also those in [8] and [11], used an integral equation for $G_{z}(x)$ derived by a procedure that goes back to Chebyshev. We use a similar equation (2.4) for the residual quantity $I_{z}(x)=$ $G_{z}(\infty)-G_{z}(x)$. There is an important difference between these equations, namely that the sum (2.5) is over "large" integers, exceeding $x / z^{2}$.

The leading term in an approximation to $I_{z}(x)$ involves the continuous function $i(s)=1-\sigma(s)$, where $\sigma$ is as in (1.9). The function $i$ has a certain adjoint $r$ defined in (3.8). The way in which these adjoints have been used in the previous literature is that the inner product $\langle i, r\rangle(s)$ (defined in (3.10)) is constant: this is used to transfer information between small values of $s$ and larger values, normally the limiting case $s \rightarrow \infty$. This device goes back to papers of de Bruijn [2]. It can, for example, be used to connect the value $\sigma(\infty)=1$ with the value of $C$ given in (1.10).

In this paper, however, we will consider the inner product of $r$ not with the continuous approximating function $i(s)$ but with the arithmetic object $i_{z}(s)=I_{z}\left(z^{s}\right)$ whose behaviour $i(s)$ will be shown to represent. The inner product $\left\langle i_{z}, r\right\rangle(s)$ will not be constant, but it will be shown in Lemma 4 to be expressible in a form which can be shown (as a consequence of Lemma 1) to be suitably small.

Finally, this estimate for the inner product can be used in a fashion analogous to that used in the continuous case. As in [6], [8] or [11] we start with information available at $s=1$. We will show how to transfer this information, using the estimate for the inner product, to larger values of $s$, thereby obtaining Theorem 1 .

A proof of a weaker version of Theorem 1 was attempted in the author's book [3], but the argument given there was incorrect. In particular, on page 270, it neglected the contribution from the terms with $n \leq x$ to a certain Stieltjes integral analogous to (3.14) below. It seems unlikely that Theorem 1 can be proved by any similar argument that does not operate on the quantity $I_{z}(x)$ (introduced in (2.2)) in some fashion, such as that employed below.

A similar oversight involving a Stieltjes integral appeared on page 58 of [3], in the course of the proof of a theorem fundamental to Theorem 1. This error (for which the present author takes responsibility) also appeared in the joint publication [5]. A correction [4] to these items was posted on the author's web pages in December 2002, but in respect of Theorem 1 this treatment was confined to providing an independent proof of a weak corollary to Theorem 1 (relating to the context $s=1$ ), which is all that was used elsewhere in [3].

Theorem 1 is of independent interest, and this paper establishes the full version, including an extension to the case when $0<\kappa<1$, and (at the cost of some extra considerations) demonstrates what the current version of the 
methods employed leads to in the context of Theorem 2. For completeness, the necessary material from [4] is included as $\S 7$.

The author thanks the referee for his comments on an earlier version of this paper.

2. An integral equation. From this point onwards we will streamline our notation somewhat by setting

$$
g_{z}(n)= \begin{cases}g(n) & \text { if } p \mid n \Rightarrow p<z \\ 0 & \text { otherwise }\end{cases}
$$

This will prevent explicit repetition of the condition on the prime $p$ expressed in (2.1). For example, (1.4) now reduces to

$$
G_{z}(x)=\sum_{1 \leq n<x} g_{z}(n) .
$$

The ideas in Lemmas 1 and 2 are best expressed in terms not of the sum defined in (1.4) but of

$$
I_{z}(x)=G_{z}(\infty)-G_{z}(x)=\sum_{n \geq x} g_{z}(n),
$$

where $g_{z}$ is as in (2.1).

The following application of Rankin's device is not new, and can be found in [10], or in the subsequent account in [3]. It also appeared earlier, in a somewhat different aspect, in Lemma 4.1 in [6].

Lemma 1. The expression defined in (2.2) satisfies

$$
0 \leq I_{z}\left(z^{s}\right) \leq G_{z}(\infty) \exp \left(-\psi_{B}(s)\right) \quad \text { if } s \geq 1,
$$

where $B$ satisfies (1.11) and $\psi_{B}$ is as in (1.12).

Since the proof is rather short, and the notations in the references just given are slightly different from that of this paper, we give a self-contained account here. It is clear from $(2.2)$ that $I_{z}(x) \geq 0$, since $g(n) \geq 0$. On the other hand, suppose $\varepsilon \geq 0$ and observe

$$
I_{z}(x) \leq \frac{1}{x^{\varepsilon}} \sum_{n \geq x} g_{z}(n) n^{\varepsilon} \leq \frac{1}{x^{\varepsilon}} \prod_{p<z}\left(1+p^{\varepsilon} g(p)\right) .
$$

Expression of $G_{z}(\infty)$ from (1.4) as a product over primes gives

$$
\begin{aligned}
\frac{I_{z}(x)}{G_{z}(\infty)} & \leq \frac{1}{x^{\varepsilon}} \prod_{p<z} \frac{1+p^{\varepsilon} g(p)}{1+g(p)}=\frac{1}{x^{\varepsilon}} \prod_{p<z}\left(1+\frac{g(p)\left(p^{\varepsilon}-1\right)}{1+g(p)}\right) \\
& \leq \frac{1}{x^{\varepsilon}} \exp \sum_{p<z} \frac{g(p)\left(p^{\varepsilon}-1\right)}{1+g(p)} .
\end{aligned}
$$


We may write $\varepsilon=c / \log z$, provided $c \geq 0$. Then when $p<z$,

$$
\frac{p^{\varepsilon}-1}{c \log p / \log z}=\frac{e^{c \log p / \log z}-1}{c \log p / \log z} \leq \frac{e^{c}-1}{c},
$$

for example because $\left(e^{x}-1\right) / x$ increases with $x$ when $x \geq 0$, as an inspection of its Maclaurin series shows. When we write $x=z^{s}$ this gives

$$
\begin{aligned}
\frac{I_{z}\left(z^{s}\right)}{G_{z}(\infty)} & \leq \frac{1}{e^{c s}} \exp \left(\frac{e^{c}-1}{\log z} \sum_{p<z} \frac{g(p)}{1+g(p)} \log p\right) \\
& \leq \exp \left(-c s+B\left(e^{c}-1\right)\right),
\end{aligned}
$$

in which (1.11) has been used.

Take $c=\log s-\log B$ when $s \geq B$ and $c=0$ otherwise, so that $c \geq 0$ as required. Lemma 1, which is trivial when $s<B$, now follows.

In the integral equation in Lemma 2 it is important that the residual sum $\Delta_{z}(s)$ is expressed as a sum involving $g_{z}(n)$ over values of $n$ that are large when $x / z$ is large, so that Lemma 1 is, in principle, applicable to these sums. In other respects the procedure in Lemma 2 is analogous to one used in [6], [8] and [11].

Lemma 2. The sum $I_{z}(x)$ defined in (2.2) satisfies

$$
I_{z}(x) \log x+\int_{x}^{\infty} I_{z}(t) \frac{d t}{t}=\kappa \int_{x / z}^{x} I_{z}(t) \frac{d t}{t}+\Delta_{z}(x)
$$

where, with $\eta$ defined as in (1.1) and (1.3),

$$
\Delta_{z}(x)=\sum_{x / z \leq m} g_{z}(m)(\eta(z)-\eta(x / m))-\sum_{p<z} g^{2}(p) \log p \sum_{\substack{k \geq x / p^{2} \\(k, p)=1}} g_{z}(k) .
$$

In this lemma, questions of convergence are trivial because $g_{z}(m)$ and $I_{z}(x)$ vanish when $m$ and $x$ are large enough.

Begin by noting that $\sum_{p \mid n} \log p=\log n$ for squarefree $n$, whence

$$
\begin{aligned}
\sum_{n \geq x} g_{z}(n) & \log n=\sum_{m p \geq x} g_{z}(m p) \log p \\
= & \sum_{m \geq x / z} g_{z}(m) \sum_{x / m \leq p<z} g(p) \log p-\sum_{m>x / z} g_{z}(m) \sum_{\substack{x / m \leq p<z \\
p \mid m}} g(p) \log p .
\end{aligned}
$$

In the last sum, arising because $g$ is supported on squarefrees, substitute 
$m=p k$. Thus

$$
\begin{aligned}
& \sum_{n \geq x} g_{z}(n) \log n \\
& \quad=\sum_{m \geq x / z} g_{z}(m) \sum_{x / m \leq p<z} g(p) \log p-\sum_{p<z} g^{2}(p) \log p \sum_{\substack{k \geq x / p^{2} \\
(k, p)=1}} g_{z}(k) .
\end{aligned}
$$

The first inner sum on the right can be expressed via (1.1) as

$$
\sum_{x / m \leq p<z} g(p) \log p= \begin{cases}\kappa \log \frac{z}{x / m}+\eta(z)-\eta(x / m) & \text { if } x / z \leq m<x, \\ \kappa \log z+\eta(z) & \text { if } m \geq x .\end{cases}
$$

The notation is streamlined by the convention (1.3), that $\eta(v)=0$ if $v<1$. This is not in conflict with (1.1), because (1.1) does not apply when $v<1$. Thus we obtain

$$
\begin{aligned}
\sum_{n \geq x} g_{z}( & n) \log n \\
=\kappa & \sum_{x / z \leq m<x} g_{z}(m) \log \frac{m}{x / z}+\kappa \sum_{m \geq x} g_{z}(m) \log z+\Delta_{z}(x),
\end{aligned}
$$

with $\Delta_{z}(x)$ as in (2.5), in which the entries $\eta(x / m)$ are null when $m \geq x$.

In the notation $(2.2)$ we have

$$
\sum_{n \geq x} g_{z}(n) \log \frac{n}{x}=\sum_{x \leq t \leq n} g_{z}(n) \frac{d t}{t}=\int_{x}^{\infty} I_{z}(t) \frac{d t}{t} .
$$

Hence, when $u \leq v$,

$$
\begin{aligned}
\int_{u}^{v} I_{z}(t) \frac{d t}{t} & =\int \sum_{\substack{u \leq t \leq v \\
t \leq m}} g_{z}(m) \frac{d t}{t} \\
& =\sum_{u \leq m<v} g_{z}(m) \log \frac{m}{u}+\sum_{m \geq v} g_{z}(m) \log \frac{v}{u} .
\end{aligned}
$$

Choose $u=x / z, v=x$ in (2.8). Then with (2.2), (2.6) and (2.7) it gives the equation (2.4) asserted in Lemma 2.

3. Adjoints and inner products. We will proceed by writing down the integral equation satisfied by a suitable continuous function $i(s)$ approximating to $I_{z}\left(z^{s}\right) / G_{z}(\infty)$. For this purpose it is better to modify our notation somewhat, as follows almost at once. 
First, however, note that (1.9) gives

$$
s \sigma^{\prime}(s)=\kappa(\sigma(s)-\sigma(s-1)) \quad \text { if } s \neq 0 .
$$

For our purposes it is preferable to note the integrated form

$$
s \sigma(s)=\int_{0}^{s} \sigma(u) d u+\kappa \int_{s-1}^{s} \sigma(u) d u \quad \text { for all } s .
$$

There is a discussion in [3] of the existence and properties of a standard solution $\sigma=\sigma_{\kappa}$, introduced in [1], satisfying

$$
\sigma(s)= \begin{cases}C s^{\kappa} & \text { if } 0 \leq s \leq 1 \\ 1+O\left(e^{-s \log s}\right) & \text { if } s>1\end{cases}
$$

where $C$ is the constant appearing in (1.10).

In $(2.4)$, set

$$
x=z^{s}, \quad t=z^{u}, \quad I_{z}(x)=i_{z}(s), \quad \Delta_{z}(x)=\delta_{z}(s),
$$

so that $s=\log x / \log z$ and $d u=d t /(t \log z)$. Then (2.4) becomes

$$
s i_{z}(s)+\int_{s}^{\infty} i_{z}(u) d u=\kappa \int_{s-1}^{s} i_{z}(u) d u+\frac{\delta_{z}(s)}{\log z} .
$$

The corresponding continuous expression appearing in our approximation to $I_{z}(x)=G_{z}(\infty)-G_{z}(s)$ will be

$$
i(s)=1-\sigma(s),
$$

so that (3.2) shows $i(s) \rightarrow 0$ as $s \rightarrow \infty$. Then (3.1) gives

$$
-s i(s)=\kappa-\int_{0}^{s} i(u) d u-\kappa \int_{s-1}^{s} i(u) d u .
$$

Because of (3.2), letting $s \rightarrow \infty$ shows

Thus

$$
\int_{0}^{\infty} i(u) d u=\kappa .
$$

$$
s i(s)+\int_{s}^{\infty} i(u) d u=\kappa \int_{s-1}^{s} i(u) d u,
$$

the equation which would result from ignoring the entry $\delta_{z}(s)$ in (3.4). Note that (3.2) and (3.5) give

$$
i(s)=1-C s^{\kappa} \quad \text { when } 0 \leq s \leq 1 .
$$

The equation (3.6) has an "adjoint"

$$
\frac{d}{d s}(s r(s))=-\kappa r(s)+\kappa r(s+1) \text {. }
$$


It is straightforward to verify that this equation has a solution in $s>0$ expressible as a Laplace transform,

$$
r(s)=\int_{0}^{\infty} \exp \left(-s x+\kappa \int_{0}^{x} \frac{1-e^{-t}}{t} d t\right) d x,
$$

for which $r(s) \sim 1 / s$ as $s \rightarrow \infty$. Observe in passing that integrating (3.7) therefore gives

$$
s r(s)-\kappa \int_{s-1}^{s} r(x+1) d x=1 .
$$

Further, define an "inner product"

$$
\langle R, r\rangle(s)=\operatorname{sr}(s) R(s)-\kappa \int_{s-1}^{s} r(x+1) R(x) d x .
$$

We can verify that $\langle\sigma, r\rangle(s)$ (and therefore $\langle i, r\rangle(s)$, from (3.5) and (3.9)) is constant, an observation leading to the consistency of the two statements in (3.2). These matters are discussed at greater length in [3]. The central idea used here is from [2].

Now write

$$
i_{z}(s)=G_{z}(\infty)\left(i(s)+\frac{\xi_{z}(s)}{\log z}\right)
$$

so that Theorem 1 asserts that $\xi_{z}(s) \leq e^{-\psi_{B}(s)+O(A)}$. The inner product $\left\langle i_{z}, r\right\rangle(s)$ (and hence $\left\langle\xi_{z}, r\right\rangle(s)$ ) will not be constant, because (3.4) contains the entry $\delta_{z}(s)$ not appearing in (3.6).

We will have more than one occasion in this paper to refer to the following lemma. One of them arises in Lemma 4, where we derive an estimate of $\left\langle\xi_{z}, r\right\rangle(s)$ needed for Theorem 1, by expressing it in terms of $\delta_{z}(s)$ and appealing to Lemma 1.

For our purposes, the definition of the Stieltjes integrals used in this paper may be taken as being supplied by integration by parts, as used in the course of the proof of Lemma 3 .

Lemma 3. Suppose that an expression $E(t)$ satisfies

$$
E(v)-E(u) \leq A \quad \text { whenever } 2 \leq u<v<z,
$$

and that an expression $f(t)$ is positive, monotone and differentiable on an interval $[x, y)$, where $2 \leq x<y<z$. Then

$$
\int_{x \leq t \leq y} f(t) d E(t)<A \max \{f(x), f(y)\} .
$$


When $f$ decreases we obtain

$$
\begin{aligned}
\int_{x \leq t \leq y} f(t) d E(t) & =\int_{x \leq t \leq y} f(t) d(E(t)-E(x)) \\
& =(E(y)-E(x)) f(y)+\int_{x}^{y}(E(t)-E(x))\left|f^{\prime}(t)\right| d t \\
& <A f(y)+A(f(x)-f(y))=A f(x) .
\end{aligned}
$$

When $f$ increases a similar argument applies, invoking $d(E(y)-E(t))$. This proves Lemma 3.

LEMMA 4. When (1.2) and (1.11) hold the inner product defined by (3.10), (3.11) and (3.8) satisfies

$$
\left\langle\xi_{z}, r\right\rangle(s) \leq \operatorname{Asr}(s) \exp \left(-\psi_{B}(s)\right) \quad \text { if } s \geq 1
$$

where $\psi_{B}$ is as in (1.12).

From $(3.11),(3.4)$ and (3.6) we obtain an integral equation for $\xi_{z}(s)$,

$$
s \xi_{z}(s)+\int_{s}^{\infty} \xi_{z}(u) d u=\kappa \int_{s-1}^{s} \xi_{z}(u) d u+\frac{\delta_{z}(s)}{G_{z}(\infty)} .
$$

From this we obtain an equation between Stieltjes integrals,

$$
\int_{s \leq u} r(u) \frac{d \delta_{z}(u)}{G_{z}(\infty)}=\int_{s \leq u} r(u)\left(u d \xi_{z}(u)-\kappa \xi_{z}(u) d u+\kappa \xi_{z}(u-1) d u\right),
$$

which we consider in two ways.

After integration by parts the first entry on the right becomes, because of $(3.7)$,

$$
\int_{s \leq u} u r(u) d \xi_{z}(u)=-s r(s) \xi_{z}(s)-\int_{s}^{\infty} \xi_{z}(u)(-\kappa r(u)+\kappa r(u+1)) d u .
$$

This gives an equation involving the inner product defined in (3.10),

$$
\begin{aligned}
\int_{s \leq u} r(u) & \frac{d \delta_{z}(u)}{G_{z}(\infty)} \\
& =-s r(s) \xi_{z}(s)+\kappa \int_{s-1}^{s} r(u+1) \xi_{z}(u) d u=-\left\langle\xi_{z}, r\right\rangle(s) .
\end{aligned}
$$

Secondly, the substitution (3.3) and the expression (2.5) for $\Delta_{z}$ give

$$
\int_{s \leq u} r(u) d \delta_{z}(u)=\int_{x \leq t} r\left(\frac{\log t}{\log z}\right) d \Delta_{z}(t)=I_{1}-I_{2},
$$


where

$$
\begin{aligned}
& I_{1}=\int_{x \leq t} r\left(\frac{\log t}{\log z}\right) d\left(\sum_{t / z \leq m} g_{z}(m)(\eta(z)-\eta(t / m))\right), \\
& I_{2}=\int_{x \leq t} r\left(\frac{\log t}{\log z}\right) d\left(\sum_{p<z} g^{2}(p) \log p \sum_{\substack{k \geq t / p^{2} \\
(k, p)=1}} g_{z}(k)\right) .
\end{aligned}
$$

Note that (3.12) and (3.13) give $G_{z}(\infty)\left\langle\xi_{z}, r\right\rangle(s)=-I_{1}+I_{2}$.

Invert the order of summation and integration in (3.14), to obtain

$$
I_{1}=\sum_{x / z \leq m} g_{z}(m) \int_{x \leq t \leq m z} r\left(\frac{\log t}{\log z}\right) d(\eta(z)-\eta(t / m)) .
$$

This step can also be checked using integration by parts, as invoked in Lemma 9 below. Here, $\eta(z)$ is independent of $t$, whence

$$
-I_{1}=\sum_{x / z \leq m} g_{z}(m) \int_{x \leq t \leq m z} r\left(\frac{\log t}{\log z}\right) d \eta(t / m) .
$$

Since $r$ decreases, (1.2) and Lemma 3 now give

$$
-I_{1} \leq A \sum_{x / z \leq m} g_{z}(m) r\left(\frac{\log x}{\log z}\right) .
$$

For the entry in (3.15) we need only observe $I_{2} \leq 0$, as follows since the $d$ expression in (3.15) is negative, the sum over $k$ decreasing as $t$ increases. (An alternative treatment of this fact appears in the proof of Lemma 9.) Now (3.12) and (3.13) lead to

$$
G_{z}(\infty)\left\langle\xi_{z}, r\right\rangle(s)=-I_{1}+I_{2} \leq A r\left(\frac{\log x}{\log z}\right) \sum_{x / z \leq m} g(m)=A r(s) I_{z}\left(z^{s-1}\right) .
$$

An application of Lemma 1 gives

$$
\left\langle\xi_{z}, r\right\rangle(s) \leq A r(s) \exp \left(-\psi_{B}(s-1)\right) .
$$

But (1.12) shows

$$
\psi_{B}(s)-\psi_{B}(s-1)=\int_{\substack{s-1<t<s \\ B<t}} \log \frac{t}{B} d t \leq \log \frac{s}{B} \leq \log s,
$$

in view of our decision in (1.11) to take $B \geq 1$. Also $\operatorname{sr}(s) \leq r(1)$, since (3.7) and (3.8) show that $\operatorname{sr}(s)$ decreases as $s$ increases. Lemma 4 follows.

4. The inductive argument. The proofs of our theorems involve a passage from the property of the inner product $\left\langle\xi_{z}, r\right\rangle(s)$ obtained in Lemma 4 to the properties required of $\xi_{z}$ itself. The argument is of a type 
used in $\S 7$ of [3], but for some of the results now stated more general versions are required. The structure of the argument is described by Lemma 6 below.

In the applications of the following lemmas the functions $Q(s)$ and $U(s)$ will differ only by continuous functions from positive or negative sums of the type $\sum_{n<z^{s}} f(n)$, so they will have only jump discontinuities, occurring where $z^{s}$ is an integer. The case of Lemma 5 in which $c \neq 0$ is not needed when $\kappa \geq 1$, but having proved it we will use it freely. A simpler version of Lemma 5 appeared in [3], in an easier context where $c=0$ and the function $Q$ could not increase at its discontinuities, this being the case relevant to the case $\kappa \geq 1$ of our Theorem 1 .

Lemma 5. Assume that $Q(s)$ is continuous in $s>0$ apart from simple jump discontinuities. Suppose that $c \geq 0, Q(s)<c s^{\kappa-1}$ when $0<s \leq 1$ and $\langle Q, r\rangle(s)<0$ when $s \geq 1$. Then $Q(s)<c s^{\kappa-1}$ for all $s>0$.

Initially we derive the case $c=0$ of Lemma 5 . If this were false, then $Q(s) \geq 0$ for some $s \geq 1$. Let $s_{1}$ be the infimum of all such $s$. Then $s_{1} \geq 1$ and $Q\left(s_{1}+\right) \geq 0$, the symbol + indicating passage to the limit as $s \rightarrow s_{1}$ through values exceeding $s_{1}$.

Note, after reference to (3.10), that the assumption on $\langle Q, r\rangle$ gives

$$
\operatorname{sr}(s) Q(s)<\kappa \int_{s-1}^{s} r(x+1) Q(x) d x \quad \text { when } s \geq 1 .
$$

If $Q$ is continuous at $s_{1}$ then taking $s=s_{1}$ gives $Q\left(s_{1}\right)<0$, a contradiction.

If $Q$ is discontinuous at $s_{1}$ then passage to the limit gives

$$
s_{1} r\left(s_{1}\right) Q\left(s_{1}+\right) \leq \kappa \int_{s_{1}-1}^{s_{1}} r(x+1) Q(x) d x \leq 0 .
$$

Hence $Q\left(s_{1}+\right) \leq 0$, so that in fact $Q\left(s_{1}+\right)=0$, since $Q\left(s_{1}+\right) \geq 0$ as already noted. Now (4.2) implies $Q(x)=0$ whenever $s_{1}-1<x<s_{1}$, contrary to the definition of $s_{1}$, so that the suggested discontinuity at $s_{1}$ cannot arise. This establishes the case $c=0$ of Lemma 5 .

To deal with the case where $c>0$ note first that when $s>1$,

$$
c \int_{s-1}^{s} r(x+1) \cdot \kappa x^{\kappa-1} d x<\operatorname{cr}(s) \int_{s-1}^{s} \kappa x^{\kappa-1} d x<\operatorname{cr}(s) s^{\kappa},
$$

since $r$ decreases. With (4.1) this shows

$$
s r(s)\left(Q(s)-c s^{\kappa-1}\right)<\kappa \int_{s-1}^{s} r(x+1)\left(Q(x)-c x^{\kappa-1}\right) d x .
$$

Define $Q^{*}(t)=Q(t)-c t^{\kappa-1}$. Then $Q^{*}$ satisfies the hypotheses previously expressed for $Q$ in the case $c=0$. The corresponding conclusion now gives $Q(s)-c s^{\kappa-1}<0$ whenever $s>0$. This establishes Lemma 5 . 
LEMMA 6. Suppose that $U_{z}(s)$ is continuous in $s>0$ apart from simple jump discontinuities, and that there is a constant $c>0$ such that $U_{z}(s) \leq$ $c s^{\kappa-1}$ when $0<s \leq 1$. Assume that $B \geq 1$ as in (1.11) and

$$
\left\langle U_{z}, r\right\rangle(s)<\operatorname{sr}(s) f(s) e^{-\psi_{B}(s)} \quad \text { when } s \geq 1 \text {, }
$$

where $\psi_{B}(s)$ is as in (1.12), $r$ satisfies (3.7), $f(s) \geq 1$, and $f(s)$ increases as $s$ increases. Then

$$
U_{z}(s) \leq f(s) e^{-\psi_{B}(s)+O(B)} \quad \text { when } s \geq 1 .
$$

Our proof of Lemma 6 invokes Lemmas 7 and 8. Lemma 7 is a variant of Lemma 4.2.8 from [3]. We give a self-contained proof as it is short. The author found the argument somewhere (possibly unpublished) in the writings of H. Iwaniec, but is currently unable to specify exactly where.

For Theorem 1, only the case $f(s)=1$ of Lemma 6 is relevant.

LEMmA 7. Suppose $U^{+}(s) \geq 0$, and that $U^{+}$is bounded and integrable on $s \geq 1$. Define $u(x)=U^{+}(x) e^{\phi(x)}$, where $\phi(x)=x \log x-D x$ for some constant $D>0$. Then

$$
\frac{\kappa}{s} \int_{s-1}^{s} U^{+}(x) d x<\frac{1}{2} e^{-\phi(s)} \sup _{s-1 \leq x \leq s} u(x),
$$

if $s / e^{D}$ exceeds a suitable constant depending on $\kappa$.

Use the fact that $\phi^{\prime}(x)=\log x+1-D$ increases with $x$. Consequently $\phi(s)-\phi(x) \leq(s-x) \phi^{\prime}(s)$ if $s-1 \leq x \leq s$. Here $u(x) \geq 0$, and the expression to be estimated in Lemma 7 does not exceed

$$
\begin{aligned}
\frac{\kappa}{s} e^{-\phi(s)} \int_{s-1}^{s} e^{\phi(s)-\phi(x)} u(x) d x & \leq \frac{\kappa}{s} e^{-\phi(s)} \int_{s-1}^{s} e^{(s-x) \phi^{\prime}(s)} d x \sup _{s-1 \leq x \leq s} u(x) \\
& \leq \frac{\kappa e^{\phi^{\prime}(s)}}{s \phi^{\prime}(s)} e^{-\phi(s)} \sup _{s-1 \leq x \leq s} u(x)
\end{aligned}
$$

provided $s>e^{D-1}$, so that $\phi^{\prime}(s)>0$. But

$$
\frac{\kappa e^{\phi^{\prime}(s)}}{s \phi^{\prime}(s)} \leq \frac{\kappa e^{1-D}}{\log s+1-D}<\frac{1}{2}
$$

if $\log s-D$ is large enough, so Lemma 7 follows. If

LEMmA 8. Suppose that $h(s)$ is bounded above on each interval $(0, S)$.

$$
h(s)<1+\frac{1}{2} \sup _{0<x \leq s} h(x) \quad \text { when } s>0,
$$

then $h(s)$ is bounded above on $s>0$.

Let $h^{*}(s)=\sup _{0<x<s} h(t)$. Then $h^{*}(s) \leq 1+\frac{1}{2} h^{*}(s)$. This says that $h^{*}(s) \leq 2$, so Lemma 8 follows. 
We now prove Lemma 6 . We may suppose $s>c_{0} B$, with a suitable constant $c_{0}$ depending on $\kappa$. For when $1 \leq s \leq c_{0} B$ we find from (1.12) that $\psi_{B}(s)=O(B)$. Thus it suffices to show that $U_{z}\left(s_{0}\right) \leq f\left(s_{0}\right) e^{O(B)}$ when $s_{0} \leq c_{0} B$, uniformly in $B$. Define $Q(s)=U_{z}(s)-c_{1} f\left(s_{0}\right)$ when $0<s \leq s_{0}$, and take $Q(s)=0$ if $s>s_{0}$. Then, referring to (3.9) and (3.10) for the calculation of the inner product, we see that by choice of $c_{1}$ with $c_{1}=e^{O(B)}$ we can ensure that for $1<s<s_{0}$,

$$
\langle Q, r\rangle(s)=\left\langle U_{z}, r\right\rangle(s)-c_{1} f\left(s_{0}\right)<s r(s) f\left(s_{0}\right) e^{O(B)}-c_{1} f\left(s_{0}\right)<0,
$$

since $\operatorname{sr}(s)$ is bounded. For $0<s<1$ we use only $Q(s)<c s^{\kappa-1}$. Then Lemma 5 gives $Q\left(s_{0}\right)<c s_{0}^{\kappa-1}$, so that

$$
U_{z}\left(s_{0}\right)<c s_{0}^{\kappa-1}+c_{1} f\left(s_{0}\right)=f\left(s_{0}\right) e^{O(B)},
$$

as was required when $1 \leq s_{0} \leq c_{0} B$.

Define $U^{+}(x)=\max \left\{U_{z}(x), 0\right\}$, so that $U^{+}(x) \geq 0$. When $s>c_{0} B$ we may argue as follows. Because of (3.10) the hypothesis of Lemma 6 gives

$$
s r(s) U_{z}(s)<\kappa \int_{s-1}^{s} U^{+}(x) r(x+1) d x+s r(s) f(s) e^{-\psi_{B}(s)} .
$$

In the integral, use $r(x+1) \leq r(s)$, to obtain

$$
s U_{z}(s) \leq \kappa \int_{s-1}^{s} U^{+}(x) d x+s f(s) e^{-\psi_{B}(s)} .
$$

We will take $D=1+\log B$ in Lemma 7 . Then $\phi(s)+B=\psi_{B}(s)$ when $s>B$ and $\psi_{B}$ is as in (1.12). Now (4.4) gives

$$
\frac{U_{z}(s)}{e^{-B}}<\frac{\kappa}{s} \int_{s-1}^{s} \frac{U^{+}(x)}{e^{-B}} d x+f(s) e^{-\phi(s)} \quad \text { when } s>B .
$$

Define $h$ by $U^{+}(s) / e^{-B}=h(s) e^{-\phi(s)} f(s)$, so that $h(s) \geq 0$. Then $u(x) / e^{-B}=h(x) f(x)$ in Lemma 7, which with (4.5) gives

$$
h(s) f(s) e^{-\phi(s)}<e^{-\phi(s)}\left(f(s)+\frac{1}{2} \sup _{s-1 \leq x \leq s} h(x) f(x)\right),
$$

provided $s>c_{2} e^{D}$, for a suitable constant $c_{2}=c_{2}(\kappa)$. But $f(x)$ increases with $x$, so this gives

$$
h(s)<1+\frac{1}{2} \sup _{s-1 \leq x \leq s} h(x),
$$

when $s>c_{2} e B$, as we have shown may be supposed in dealing with Lemma 6 .

With Lemma 8 , this gives $U^{+}(s) \ll e^{B-\phi(s)} f(s)$ when $s>c_{0} B$. Since $U_{z}(s) \leq U^{+}(s)$, this completes the proof of Lemma 6 . 
5. The proof of Theorem 1. As previously indicated, the proof is of an inductive type. It begins by using the fact that the case $s=1$ of the theorem is already known. We discuss the proof of this special case in the Appendix.

The use of Lemma 6 when $s$ is close to 1 requires knowledge of the situation in which $0<s \leq 1$. Note from (1.4) that $G_{z}(w)=G_{w}(w)$ when $w<z$. We will write $w=z^{s}$ later. These considerations lead to

$$
\frac{G_{z}(w)}{G_{w}(\infty)}=\frac{G_{w}(w)}{G_{w}(\infty)} \geq C\left(1-O\left(\frac{A}{\log w}\right)\right) \quad \text { if } 2 \leq w \leq z
$$

Here $C=\sigma(1)$ is as described in (1.10).

We will need the relation between $G_{w}(\infty)$ and $G_{z}(\infty)$. In fact, since $w \leq z$, we may use

$$
\frac{G_{z}(\infty)}{G_{w}(\infty)}=\prod_{w \leq p<z}(1+g(p))=\exp \sum_{w \leq p<z} \log (1+g(p)) .
$$

When (1.2) holds we may apply Lemma 3 with $E(t)=\eta(t)$, to obtain

$$
\sum_{w \leq p<z} g(p)=\int_{w \leq t<z} \frac{1}{\log t} d(\kappa \log t+\eta(t)) \leq \kappa \log \left(\frac{\log z}{\log w}\right)+\frac{A}{\log w} .
$$

Now (5.2) gives

$$
\frac{G_{z}(\infty)}{G_{w}(\infty)} \leq \exp \sum_{w \leq p<z} g(p) \leq\left(\frac{\log z}{\log w}\right)^{\kappa} \exp \left(O\left(\frac{A}{\log w}\right)\right) .
$$

If $w \geq e^{c A}$ with $c$ sufficiently large this gives

$$
\frac{G_{w}(\infty)}{G_{z}(\infty)} \geq\left(\frac{\log w}{\log z}\right)^{\kappa}\left(1-O\left(\frac{A}{\log w}\right)\right) .
$$

If $w<e^{c A}$ then this inequality follows trivially since $G_{w}(\infty) / G_{z}(\infty) \geq 0$.

With (5.1) this gives, when we write $w=z^{s}$, so that $0<s \leq 1$,

$$
\frac{G_{z}\left(z^{s}\right)}{G_{z}(\infty)} \geq C s^{\kappa}\left(1-O\left(\frac{A}{s \log z}\right)\right) \geq \sigma(s)-O\left(\frac{A s^{\kappa-1}}{\log z}\right) .
$$

Thus $i_{z}(s) \leq i(s)+c A s^{\kappa-1} / \log z$ for some constant $c$. This says that $\xi_{z}(s) \leq$ $c A s^{\kappa-1}$ when $0<s \leq 1$. The $A$-dependence here is quite good, but this feature fades at the next step.

Apply Lemma 6 with $U_{z}(s)=\xi_{z}(s) / A$. Lemma 4 shows that (4.3) applies with $f(s)=1$, so that Lemma 6 gives

$$
\xi_{z}(s) \leq e^{-\psi_{B}(s)+O(B)} \quad \text { when } s \geq 1 .
$$

But $B=O(A)$ in the context of Theorem $1, \xi_{z}$ is as in (3.11) and $i(s)$ is as in (3.5), so Theorem 1 follows. 
6. The proof of Theorem 2. The two-sided nature of this theorem is perhaps more familiar, but its proof involves some additional complications. In the first place we replace Lemma 4 by the following analogue.

Lemma 9. When (1.5) and (1.11) hold the inner product defined by (3.10), (3.11) and (3.8) satisfies

$$
\left|\left\langle\xi_{z}, r\right\rangle(s)\right| \leq 2 A s^{2} r(s) \exp \left(-\psi_{B}(s)\right) \quad \text { if } s \geq 1,
$$

where $\psi_{B}$ is as in (1.12).

The two-sided version of Lemma 3, which can be deduced by also applying it to $-E$, is that under the additional hypothesis

$$
|E(v)-E(u)| \leq A \quad \text { whenever } 2 \leq u<v<z
$$

we find

$$
\left|\int_{x \leq t \leq y} f(t) d E(t)\right|<A \max \{f(x), f(y)\} .
$$

Proceed as in Lemma 4 as far as (3.16). Then (1.11) implies that in this way we obtain

$$
\left|I_{1}\right| \leq A \sum_{x / z \leq m} g_{z}(m) r\left(\frac{\log x}{\log z}\right) .
$$

To estimate $I_{2}$ integrate (3.15) by parts, to obtain

$$
\begin{aligned}
I_{2}= & \sum_{p<z} g^{2}(p) \log p\left\{-r\left(\frac{\log x}{\log z}\right) \sum_{\substack{k \geq x / p^{2} \\
(k, p)=1}} g_{z}(k)\right. \\
& \left.+\int_{x \leq t} \sum_{\substack{k \geq t / p^{2} \\
(k, p)=1}} g_{z}(k)\left|d r\left(\frac{\log t}{\log z}\right)\right|\right\} .
\end{aligned}
$$

Here, the integral (arising with a positive coefficient because $r$ decreases) lies between 0 and

$$
\sum_{\substack{k \geq x / p^{2} \\(k, p)=1}} g_{z}(k) \int_{x \leq t}\left|d r\left(\frac{\log t}{\log z}\right)\right|,
$$

in which we replaced the sum over $k$ by its maximum value. Thus

$$
0 \geq I_{2} \geq-\sum_{p<z} g^{2}(p) \log p \sum_{\substack{k \geq x / p^{2} \\(k, p)=1}} g_{z}(k) r\left(\frac{\log x}{\log z}\right) .
$$

Now (1.6) gives

$$
\left|I_{2}\right| \leq A r\left(\frac{\log x}{\log z}\right) \sum_{k \geq x / z^{2}} g_{z}(k)
$$


We complete the proof of Lemma 9 by arguing as with Lemma 4 .

Continue by quoting the fact that the case $s=1$ of Theorem 2 was, in essence, established as Lemma 5.4 of [6]. Actually the argument in [6] started from a formulation slightly different from (1.1), but the effect of starting from (1.1) is to make the inference slightly simpler. Also, in [6] the assumption corresponding to (1.5) was of the form $-L<\eta(v)-\eta(u)<A$ when $1<u<v$, and only the $L$-dependence of the $O$-constants was made explicit. It is easy to check that the argument from [6] leads to the second of the two statements in (6.1). A somewhat different presentation along the lines of the appendix to this paper is also possible.

In place of (5.1) we now infer

$$
\frac{G_{z}(w)}{G_{w}(\infty)}=\frac{G_{w}(w)}{G_{w}(\infty)}=C\left(1+O\left(\frac{A}{\log w}\right)\right) \quad \text { if } 2 \leq w \leq z .
$$

For the relation between $G_{w}(\infty)$ and $G_{z}(\infty)$, use

$$
\begin{aligned}
\frac{G_{z}(\infty)}{G_{w}(\infty)} & =\prod_{w \leq p<z}(1+g(p))=\exp \sum_{w \leq p<z} \log (1+g(p)) \\
& =\exp \sum_{w \leq p<z}\left(g(p)+O\left(g^{2}(p)\right)\right) .
\end{aligned}
$$

A partial summation from (1.1) and (1.5) gives

$$
\sum_{w \leq p<z} g(p)=\int_{w<t \leq z} \frac{1}{\log t} d(\kappa \log t+O(A))=\kappa \log \left(\frac{\log z}{\log w}\right)+O\left(\frac{A}{\log w}\right) .
$$

Proceeding from (1.6) we can infer

$$
\sum_{w \leq p<z} g^{2}(p) \leq \frac{1}{\log w} \sum_{w \leq p<z} g^{2}(p) \log p \leq \frac{A}{\log w} .
$$

Define $\alpha=c e^{A}$ with $c$ sufficiently large. Suppose first that $w \geq \alpha$. Then the use of (6.2) gives, when $z>w$,

$$
\frac{G_{z}(\infty)}{G_{w}(\infty)}=\left(\frac{\log z}{\log w}\right)^{\kappa} \exp \left(O\left(\frac{A}{\log w}\right)\right) .
$$

This implies the case in which $z>w \geq \alpha$ of

$$
\frac{G_{w}(\infty)}{G_{z}(\infty)}=\left(\frac{\log w}{\log z}\right)^{\kappa}\left(1+O\left(\frac{e^{O(A)}}{\log w}\right)\right) \quad \text { when } 2 \leq w<z,
$$

the outer $O$-symbols allowing the terms in question to have either sign. To obtain (6.3) when $2 \leq w<\alpha$, use a first-order version of (6.2) to find

$$
\frac{G_{w}(\infty)}{G_{\alpha}(\infty)}=\exp \sum_{w \leq p<\alpha} O(g(p))=\left(\frac{\log \alpha}{\log z}\right)^{O(1)} e^{O(A)}=e^{O(A)} .
$$


With the case $w=\alpha$ of $(6.3)$ this gives

$$
G_{w}(\infty) / G_{z}(\infty)=e^{O(A)} / \log ^{\kappa} z \quad \text { when } 2 \leq w<\alpha .
$$

Hence (6.3) is valid whenever $2 \leq w<z$, since if $z<\alpha$ it follows after replacing $\alpha$ by $z$ in (6.4).

Now write $w=z^{s}$ and use (6.1) and (6.3). This gives, for $0<s \leq 1$,

$$
\frac{G_{z}\left(z^{s}\right)}{G_{z}(\infty)}=C s^{\kappa}\left(1+O\left(\frac{e^{O(A)}}{s \log z}\right)\right)=\sigma(s)+O\left(\frac{e^{O(A)} s^{\kappa-1}}{\log z}\right) .
$$

The $A$-dependence, arising from considerations relating to small values of $w$ which appear in this argument, is not very good, but it is not worse than those arising elsewhere from our use of Lemmas 7 and 6 .

In the notation of (2.2) and (3.3), this gives

$$
\frac{i_{z}(s)}{G_{z}(\infty)}=i(s)+O\left(\frac{e^{O(A)} s^{\kappa-1}}{\log z}\right) \quad \text { when } 0<s \leq 1,
$$

with $i(s)=1-\sigma(s)$ as in (3.5). Thus, in the notation (3.11), we have found there is a constant $c_{1}$ such that $\left|\xi_{z}(s)\right| \leq e^{c_{1} A} s^{\kappa-1}$ when $0<s \leq 1$.

Now apply Lemma 6 , twice, with $U_{z}(s)= \pm \xi_{z}(s) / e^{c_{1} A}$. Lemma 4 shows that (4.3) holds with $f(s)=s$, because $r(s)=O(1 / s)$. Thus in either case the conditions of Lemma 6 are satisfied. This process yields the twosided inequality $\left|\xi_{z}(s)\right| \leq s e^{-\psi_{B}(s)+O(A+B)}$. Via (3.11) this leads to Theorem 2 .

7. Appendix. The preceding treatment required separate proofs of Theorems 1 and 2 at the point $s=1$. In principle, these were provided in [9] and [6] respectively, in the former case making use of the result from [6] and stating a result slightly weaker than that used in this paper. For this, one may use the more direct method given in [5], also described in [3], save that these accounts are incomplete. They can be corrected as follows. We need to assume (1.2), which is stronger than the hypothesis stated in [3] and $[5]$.

The text in [5] refers to

$$
\begin{aligned}
E_{n}(y) & =\sum_{p<y} g(p) \log p-\sum_{\substack{p<\sqrt{y} \\
(p, n)=1}} g^{2}(p) \log p-\kappa \log y \\
& =\eta(y)-\sum_{\substack{p<\sqrt{y} \\
(p, n)=1}} g^{2}(p) \log p,
\end{aligned}
$$


in which (1.1) has been used. The identity

$$
G(x) \log x-(\kappa+1) \int_{1}^{x} G(t) \frac{d t}{t}=\delta(x)=\sum_{n<x} g(n) E_{n}\left(\frac{x}{n}\right),
$$

is obtained as in [6] (or see [5] or [3]), by the process used to derive Lemma 2 in this paper.

When $u \leq v$ the hypothesis (1.2) gives

$$
E_{n}(v)-E_{n}(u)=\eta(v)-\eta(u)-\sum_{\substack{\sqrt{u} \leq p<\sqrt{v} \\ p \nmid n}} g^{2}(p) \log p \leq A .
$$

Consequently Lemma 3 may be used with $E(t)=E_{n}(t / n)$.

The corresponding discussion in [3] was more involved because it initially referred to $G_{z}(x)$ rather than to $G(x)=G_{x}(x)$, the specialisation to the case $z=x$ not having been made at that stage.

In [3] and [5] the procedure is to work with $H(x)=G(x) / \log ^{\kappa} x$, and to aim to establish an inequality related to Cauchy's convergence criterion,

$$
H(y)-H(x) \leq \frac{c A}{\log x} \sup _{x \leq t<y} H(t),
$$

for some absolute constant $c$. The case $s=1$ of Theorem 1 would then follow as in [3] or [5].

Because (7.1) gives

$$
\int_{2}^{x} \frac{d \delta(t)}{\log ^{\kappa+1} t}=\int_{2}^{x} \frac{1}{\log ^{\kappa+1} t}\left(\log t d G(t)-\kappa \frac{G(t)}{t} d t\right)=\int_{2}^{x} d \frac{G(t)}{\log ^{\kappa} t},
$$

the equation (7.1) integrates to

$$
H(y)-H(x)=\int_{x}^{y} \frac{d \delta(t)}{\log ^{\kappa+1} t}=\int_{x}^{y} \frac{1}{\log ^{\kappa+1} t} d \sum_{1 \leq n<t} g(n) E_{n}\left(\frac{t}{n}\right) .
$$

The argument in [3] and [5] ignored the contribution to $H(y)-H(x)$ arising from those $g(n)$ with $n<x$. Since $g(n)$ is independent of $t$, this contribution is

$$
\begin{aligned}
\int_{x \leq t<y} \frac{1}{\log ^{\kappa+1} t} d \sum_{1 \leq n<x} g(n) E_{n}\left(\frac{t}{n}\right) & =\sum_{1 \leq n<x} g(n) \int_{x \leq t<y} \frac{1}{\log ^{\kappa+1} t} d E_{n}\left(\frac{t}{n}\right) \\
& \leq \sum_{1 \leq n<x} g(n) \frac{A}{\log ^{\kappa+1} x}=\frac{A H(x)}{\log x},
\end{aligned}
$$

by (7.2) and Lemma 3. In conjunction with the treatment in [3] or [5] of the terms with $n>x$ this gives the required estimate (7.3). 
It is possible to give a treatment of the case $s=1$ of Theorem 2 along similar lines, in which the passage from the identity (7.1) to the required result is somewhat different from that in [6].

\section{References}

[1] N. C. Ankeny and H. Onishi, The general sieve, Acta Arith. 10 (1964/1965), 31-62.

[2] N. G. de Bruijn, On the number of uncancelled elements in the sieve of Eratosthenes, Koninkl. Nederl. Akad. Wetensch. Proc. Sect. Sci. 53 (1950), 803-812 = Indag. Math. 12 (1950), 247-256.

[3] G. Greaves, Sieves in Number Theory, Ergeb. Math. Grenzgeb. (3) 43, Springer, Berlin, 2001.

[4] —, http://www.cardiff.ac.uk/maths/people/greaves_research.html

[5] G. Greaves and M. Huxley, One-sided sifting density hypotheses in Selberg's sieve, in: Number Theory (Turku, 1999), M. Jutila and T. Metsänkylä (eds.), de Gruyter, Berlin, 2001, 105-114.

[6] H. Halberstam and H.-E. Richert, Sieve Methods, London Math. Soc. Monogr. 4, Academic Press, London, 1974.

[7] A. Hildebrand, The asymptotic behavior of the solutions of a class of differentialdifference equations, J. London Math. Soc. (2) 42 (1990), 11-31.

[8] - On the number of positive integers $\leq x$ and free of prime factors $>y$, J. Number Theory 22 (1986), 289-307.

[9] D. A. Rawsthorne, Selberg's sieve estimate with a one sided hypothesis, Acta Arith. 41 (1982), 281-289.

[10] A. Selberg, Collected Papers, Vol. II, Springer, Berlin, 1991.

[11] J. M. Song, Sums of nonnegative multiplicative functions over integers without large prime factors I, Acta Arith. 97 (2001), 329-351.

[12] - Sums of nonnegative multiplicative functions over integers without large prime factors II, ibid. 102 (2002), 105-129.

[13] G. Tenenbaum, Note on a paper by Joung Min Song, ibid. 97 (2001), 353-360.

[14] G. Tenenbaum et J. Wu, Moyennes de certaines fonctions multiplicatives sur les entiers friables, J. Reine Angew. Math. 564 (2003), 119-166.

School of Mathematics

Cardiff University

Senghennydd Road

Cardiff CF24 4AG

Wales, U.K.

E-mail: Greaves@Cardiff.ac.uk

Received on 26.7.2004

and in revised form on 22.3.2005 